\title{
Calmness of efficient solution maps in parametric vector optimization*
}

\author{
T. D. Chuong $\dagger$ A. Y. Kruger ${ }^{\ddagger}$ and J.-C. Yao ${ }^{\S}$ \\ January 7, 2011
}

\begin{abstract}
The paper is concerned with the stability theory of the efficient solution map of a parametric vector optimization problem. Utilizing the advanced tools of modern variational analysis and generalized differentiation, we study the calmness of the efficient solution map. More explicitly, new sufficient conditions in terms of the Fréchet and limiting coderivatives of parametric multifunctions for this efficient solution map to have the calmness at a given point in its graph are established by employing the approach of implicit multifunctions. Examples are also provided for analyzing and illustrating the results obtained.
\end{abstract}

Key words. Parametric vector optimization, efficient solution map, calmness, coderivative, implicit multifunction.

AMS subject classifications. 49K40, 49J52, 90C29, 90C31.

${ }^{*}$ This work was supported by the National Science Council of ROC under grant NSC 992221-E-110-038-MY3, the Australian Research Council Grant DP110102011 and a grant from the NAFOSTED (Vietnam).

$\dagger$ Department of Mathematics, Dong Thap University, Cao Lanh City, Dong Thap Province, Vietnam; email: chuongthaidoan@yahoo.com.

${ }^{\ddagger}$ Centre for Informatics and Applied Optimization, Graduate School of Information Technology \& Mathematical Sciences, University of Ballarat, P.O. Box 663, Ballarat, Vic. 3353, Australia; email: akruger@ballarat.edu.au.

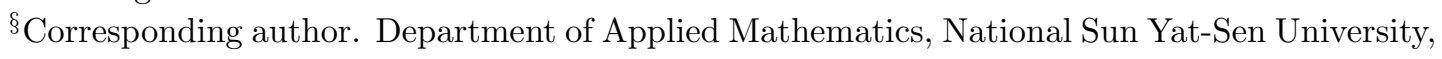
Kaohsiung 804, Taiwan; email: yaojc@math.nsysu.edu.tw. 


\section{Introduction}

The paper mainly deals with the stability theory of parametric vector optimization problems. We first give some notation and definitions.

Let $f: P \times X \rightarrow Y$ be a vector function, where $X$ and $Y$ are Banach spaces, and $P$ is a metric space. Let $K \subset Y$ be a pointed, closed and convex cone with apex at the origin.

Definition 1.1 We say that $y \in A$ is an efficient point of a subset $A \subset Y$ with respect to $K$ if and only if $(y-K) \cap A=\{y\}$. The set of efficient points of $A$ is denoted by $\operatorname{Eff}_{K} A$. We stipulate that $\operatorname{Eff}_{K} \emptyset=\emptyset$.

We consider the following parametric vector optimization problem:

$$
\text { Find } \operatorname{Eff}_{K}\{f(p, x) \mid x \in X\}
$$

where $x$ is the unknown (decision variable) and $p \in P$ a parameter.

For each $p \in P$, we put

$$
\begin{gathered}
\mathcal{F}(p):=\operatorname{Eff}_{K}\{f(p, x) \mid x \in X\} \\
\mathcal{S}(p):=\{x \in X \mid f(p, x) \in \mathcal{F}(p)\},
\end{gathered}
$$

and call $\mathcal{F}: P \rightrightarrows Y$ and $\mathcal{S}: P \rightrightarrows X$ the efficient point multifunction and efficient solution map of (1.1) respectively. $x \in \mathcal{S}(p)$, that is, $f(p, x) \in \mathcal{F}(p)$ indicates that $x$ is an efficient solution of (1.1).

Stability analysis in vector optimization has attracted considerable attention of many researchers. One of the main problems here is to find sufficient conditions for the efficient solution map $\mathcal{S}$ to have certain stability properties. We refer the

reader to $[1-3,5-10,12,13,23,28-30]$ and the references therein for more details and discussions.

A multifunction $F: P \rightrightarrows Y$ from a metric space $(P, d)$ into a Banach space $Y$ is calm at a given point $(\bar{p}, \bar{y})$ of its graph (see, e.g., $[24,26]$ ) if there exist neighborhoods $U$ of $\bar{p}, V$ of $\bar{y}$ and a real number $\ell>0$ such that

$$
F(p) \cap V \subset F(\bar{p})+\ell d(p, \bar{p}) B_{Y} \quad \forall p \in U,
$$

where $B_{Y}$ denotes the closed unit ball of $Y$. With $V:=Y$, calmness reduces to the local upper Lipschitzian property of multifunctions, introduced by Robinson [27]. 
Calmness of multifunctions plays an important role in many issues of mathematical programming like optimality conditions, error bounds, weak sharp minima, metric subregularity, or stability of solutions. The reader is referred to [14-16,19,31,32] and the references therein for various characterizations and applications.

In this paper we use advanced tools of modern variational analysis and generalized differentiation to study the calmness of the efficient solution map $\mathcal{S}$ of (1.1). More precisely, new sufficient conditions in terms of the Fréchet and limiting (Mordukhovich) coderivatives [24] for the efficient solution map $\mathcal{S}$ to be calm at a given point in its graph are established by using the approach of implicit multifunctions (see $[17,21,22])$. Our approach is based on a technique of implicit multifunctions which involves the Ekeland variational principle [11] and the fuzzy sum rule for Fréchet subdifferentials [24].

The rest of the paper is organized as follows. In Section 2, we first provide the basic definitions and notation from variational analysis and set-valued analysis. Then we recall some known auxiliary results which will be useful hereafter. The main results will be presented in Section 3 where we first provide sufficient conditions for an implicit multifunction to be calm at a given point and then derive the calmness of the efficient solution map $\mathcal{S}$ of (1.1) by means of exploiting these implicit multifunction results. Moreover, examples are provided to illustrate the results.

\section{Preliminaries and auxiliary results}

Throughout the paper we use the standard notation of variational analysis and generalized differentiation; see, e.g., [24,26]. Unless otherwise stated, all spaces under consideration are Banach spaces whose norms are always denoted by $\|\cdot\|$. The canonical pairing between $X$ and its topological dual $X^{*}$ is denoted by $\langle\cdot, \cdot\rangle$. In this setting, $w^{*}$ denotes the weak* topology in $X^{*}$, and $A^{*}$ denotes the adjoint operator of a linear continuous operator $A$. The symbols $B_{X}$ and $B_{X^{*}}$ stand for the closed unit balls of $X$ and $X^{*}$, respectively. The closed ball with center $x$ and radius $\rho$ is denoted by $B_{\rho}(x)$. As usual, the distance from $u \in X$ to $\Omega \subset X$ is denoted by $\operatorname{dist}(u, \Omega):=\inf _{x \in \Omega}\|x-u\|$.

Given a set-valued mapping $F: X \rightrightarrows X^{*}$ between a Banach space $X$ and its topological dual $X^{*}$, we denote by

$$
\begin{aligned}
\operatorname{Limsup}_{x \rightarrow \bar{x}} F(x):=\left\{x^{*} \in X^{*} \mid\right. & \exists \text { sequences } x_{k} \rightarrow \bar{x} \text { and } x_{k}^{*} \stackrel{w^{*}}{\longrightarrow} x^{*} \\
& \text { with } \left.x_{k}^{*} \in F\left(x_{k}\right) \text { for all } k \in \mathbb{N}\right\}
\end{aligned}
$$


the sequential Painlevé-Kuratowski upper/outer limit with respect to the norm topology of $X$ and the weak ${ }^{*}$ topology of $X^{*}$, where $\mathbb{N}:=\{1,2, \ldots\}$.

Given $\Omega \subset X$ and $\varepsilon \geq 0$, define the collection of $\varepsilon$-normals to $\Omega$ at $\bar{x} \in \Omega$ by

$$
\widehat{N}_{\varepsilon}(\bar{x} ; \Omega):=\left\{x^{*} \in X^{*} \mid \limsup _{x \Omega \bar{x}} \frac{\left\langle x^{*}, x-\bar{x}\right\rangle}{\|x-\bar{x}\|} \leq \varepsilon\right\},
$$

where $x \stackrel{\Omega}{\rightarrow} \bar{x}$ means that $x \rightarrow \bar{x}$ with $x \in \Omega$. When $\varepsilon=0$, the set $\widehat{N}(\bar{x} ; \Omega):=$ $\widehat{N}_{0}(\bar{x} ; \Omega)$ in $(2.1)$ is a cone called Fréchet normal cone to $\Omega$ at $\bar{x}$.

The limiting (Mordukhovich) normal cone $N(\bar{x} ; \Omega)$ is obtained from $\widehat{N}_{\varepsilon}(x ; \Omega)$ by taking the sequential Painlevé-Kuratowski upper limit in the weak* topology of $X^{*}$ as

$$
N(\bar{x} ; \Omega):=\operatorname{Limsup}_{\substack{x \frac{\Omega}{\varepsilon \downarrow 0} \bar{x} \\ \varepsilon}} \widehat{N}_{\varepsilon}(x ; \Omega),
$$

where one can put $\varepsilon=0$ when $\Omega$ is closed around $\bar{x}$ and the space $X$ is Asplund, i.e., a Banach space whose separable subspaces have separable duals. Here, $\Omega$ is said to be (locally) closed around $\bar{x}$ if there is a closed neighborhood $U$ of $\bar{x}$ such that $\Omega \cap U$ is closed. It is well-known that the class of Asplund spaces is sufficiently large containing, in particular, all reflexive spaces and all spaces with separable duals. We refer the readers to the books $[24,25]$ for numerous characterizations and discussions of Asplund spaces.

Let $F: X \rightrightarrows Y$ be a set-valued mapping between Banach spaces with the domain $\operatorname{dom} F:=\{x \in X \mid F(x) \neq \emptyset\}$ and graph $\operatorname{gph} F:=\{(x, y) \in X \times Y \mid y \in F(x)\}$. We will assume that $X \times Y$ is equipped with a norm compatible with the norms in $X$ and $Y$, e.g., $\|(x, y)\|=\max (\|x\|,\|y\|)$.

The Fréchet and limiting (Mordukhovich) coderivatives of $F$ at $(\bar{x}, \bar{y}) \in \operatorname{gph} F$ are defined respectively by

$$
\begin{array}{ll}
\widehat{D}^{*} F(\bar{x}, \bar{y})\left(y^{*}\right):=\left\{x^{*} \in X^{*} \mid\left(x^{*},-y^{*}\right) \in \widehat{N}((\bar{x}, \bar{y}) ; \operatorname{gph} F)\right\} & \forall y^{*} \in Y^{*}, \\
D^{*} F(\bar{x}, \bar{y})\left(y^{*}\right):=\left\{x^{*} \in X^{*} \mid\left(x^{*},-y^{*}\right) \in N((\bar{x}, \bar{y}) ; \operatorname{gph} F)\right\} & \forall y^{*} \in Y^{*}
\end{array}
$$

In other words,

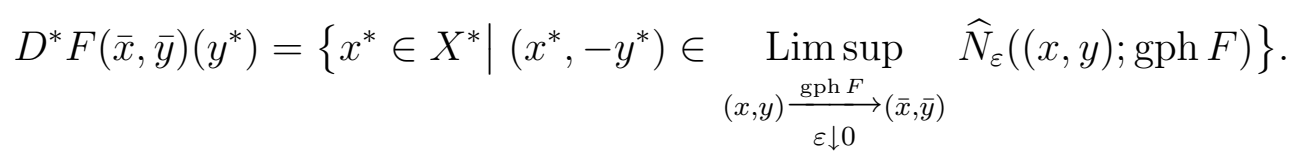


Extending the finite dimensional definition of Ioffe and Outrata [18], we define the outer coderivative of $F$ at $(\bar{x}, \bar{y})$ by the formula

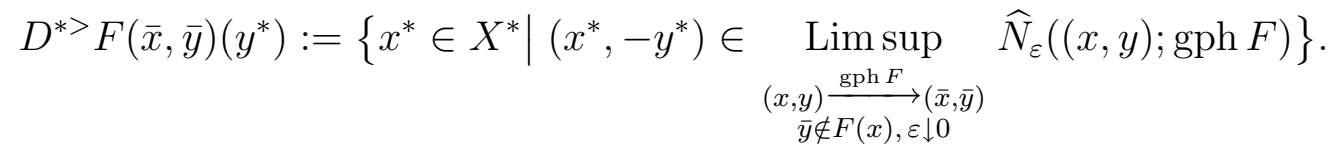

If $F$ is a single-valued mapping, to simplify the notation, one writes $\widehat{D}^{*} F(\bar{x})\left(y^{*}\right)$, $D^{*} F(\bar{x})\left(y^{*}\right)$, and $D^{*>} F(\bar{x})\left(y^{*}\right)$ instead of $\left.\widehat{D}^{*} F(\bar{x}, F(\bar{x}))\left(y^{*}\right)\right), D^{*} F(\bar{x}, F(\bar{x}))\left(y^{*}\right)$, and $D^{*>} F(\bar{x}, F(\bar{x}))\left(y^{*}\right)$ respectively.

If a single-valued mapping $f: X \rightarrow Y$ is Fréchet differentiable at $\bar{x}$ (with the derivative $\nabla f(\bar{x}))$ then $\widehat{D}^{*} f(\bar{x})\left(y^{*}\right)=\left\{(\nabla f(\bar{x}))^{*} y^{*}\right\}$ for any $y^{*} \in Y^{*}$. If, additionally, the derivative is strict, that is, (see e.g. [24])

$$
\lim _{x, u \rightarrow \bar{x}} \frac{f(x)-f(u)-\langle\nabla f(\bar{x}), x-u\rangle}{\|x-u\|}=0,
$$

then $D^{*} f(\bar{x})\left(y^{*}\right)=\left\{(\nabla f(\bar{x}))^{*} y^{*}\right\}$. Thus, the limiting coderivative is a generalization of the adjoint operator to the strict derivative; see [24] for more details.

For an extended real-valued function $\varphi: X \rightarrow \overline{\mathbb{R}}:=[-\infty, \infty]$, we define its domain $\operatorname{dom} \varphi=\{x \in X \mid \varphi(x)<\infty\}$ and epigraph epi $\varphi=\{(x, \mu) \in X \times \mathbb{R} \mid \mu \geq$ $\varphi(x)\}$. The Fréchet and limiting subdifferentials of $\varphi$ at $\bar{x}$ with $|\varphi(\bar{x})|<\infty$ are defined respectively by

$$
\begin{aligned}
& \widehat{\partial} \varphi(\bar{x}):=\left\{x^{*} \in X^{*} \mid\left(x^{*},-1\right) \in \widehat{N}((\bar{x}, \varphi(\bar{x})) ; \operatorname{epi} \varphi)\right\} \\
& \partial \varphi(\bar{x}):=\left\{x^{*} \in X^{*} \mid\left(x^{*},-1\right) \in N((\bar{x}, \varphi(\bar{x})) ; \operatorname{epi} \varphi)\right\} .
\end{aligned}
$$

If $|\varphi(\bar{x})|=\infty$, then one puts $\widehat{\partial} \varphi(\bar{x})=\partial \varphi(\bar{x})=\emptyset$.

Obviously, $\widehat{\partial} \varphi(\bar{x}) \subset \partial \varphi(\bar{x})$, and $0 \in \widehat{\partial} \varphi(\bar{x})$ if $\bar{x}$ is a local minimum of $\varphi$ (the nonsmooth version of Fermat's rule, see, e.g., [24, Proposition 1.114]).

Extending [24, Definition 1.67 (ii)], we say that $F^{-1}: Y \rightarrow X$ is partially outer sequentially normally compact at $(\bar{y}, \bar{x}) \in \operatorname{gph}\left(F^{-1}\right)$ if for any sequence $\left(\varepsilon_{k}, x_{k}, y_{k}, x_{k}^{*}, y_{k}^{*}\right) \in[0, \infty) \times(\operatorname{gph} F) \times X^{*} \times Y^{*}$ satisfying

$$
\begin{gathered}
\varepsilon_{k} \downarrow 0,\left(x_{k}, y_{k}\right) \stackrel{\operatorname{gph} F}{\longrightarrow}(\bar{x}, \bar{y}), x_{k} \notin F^{-1}(\bar{y}),\left(x_{k}^{*}, y_{k}^{*}\right) \in \widehat{N}_{\varepsilon_{k}}\left(\left(x_{k}, y_{k}\right), \operatorname{gph} F\right), \\
\left\|x_{k}^{*}\right\| \rightarrow 0, \quad \text { and } y_{k}^{*} \stackrel{w^{*}}{\longrightarrow} 0
\end{gathered}
$$

one has $\left\|y_{k}^{*}\right\| \rightarrow 0$ as $k \rightarrow \infty$. This property is satisfied automatically if $\operatorname{dim} Y<\infty$.

The following fuzzy sum rule for Fréchet subdifferentials will be useful in the sequel (see [24, Lemma 2.32] or [20, Rule 2.2]). 
Lemma 2.1 Let $X$ be an Asplund space. Suppose that $\varphi_{1}: X \rightarrow \mathbb{R}$ is Lipschitz continuous around $\bar{x} \in X$ and $\varphi_{2}: X \rightarrow \overline{\mathbb{R}}$ is finite at $\bar{x}$ and l.s.c. around this point. Then for any $\varepsilon>0$ there are $x_{i} \in \bar{x}+\varepsilon B_{X}$ with $\left|\varphi_{i}\left(x_{i}\right)-\varphi_{i}(\bar{x})\right| \leq \varepsilon, i=1,2$, such that $\widehat{\partial}\left(\varphi_{1}+\varphi_{2}\right)(\bar{x}) \subset \widehat{\partial} \varphi_{1}\left(x_{1}\right)+\widehat{\partial} \varphi_{2}\left(x_{2}\right)+\varepsilon B_{X^{*}}$.

In what follows we also use the Ekeland variational principle [11].

Lemma 2.2 (Ekeland Variational Principle). Let $(X, d)$ be a complete metric space and $f: X \rightarrow \overline{\mathbb{R}}$ be a proper l.s.c. function bounded from below. Let $\varepsilon>0$ and $x_{0} \in X$ be given such that $f\left(x_{0}\right) \leq \inf _{x \in X} f(x)+\varepsilon$. Then for any $\lambda>0$ there is $\bar{x} \in X$ satisfying

(i) $f(\bar{x}) \leq f\left(x_{0}\right)$,

(ii) $d\left(\bar{x}, x_{0}\right) \leq \lambda$,

(iii) $f(\bar{x})<f(x)+\frac{\varepsilon}{\lambda} d(x, \bar{x})$ for all $x \in X \backslash\{\bar{x}\}$.

\section{Calmness of the efficient solution map}

In this section we shall provide new sufficient conditions for the efficient solution map $\mathcal{S}$ of (1.1) to be calm at a reference point in its graph. To do this, we first need to establish the calmness of an implicit multifunction at a given point.

Let $X, Y$ and $P$ be spaces as stated above and $F: P \times X \rightrightarrows Y$ be a parametric multifunction. By means of this parametric multifunction one can define an implicit multifunction $G: P \rightrightarrows X$ as follows:

$$
G(p):=\{x \in X \mid 0 \in F(p, x)\}
$$

We now give sufficient conditions for the implicit multifunction $G$ to have the calmness property at a given point. In what follows we use the notation $F_{p}:=F(p, \cdot)$, $p \in P$. For $(\bar{x}, \bar{y}) \in \operatorname{gph} F_{\bar{p}}$ define

$$
\begin{aligned}
c\left[F_{\bar{p}}\right](\bar{x}, \bar{y}):=\lim _{\delta \downarrow 0} \inf \left\{\left\|x^{*}\right\| \mid x^{*}\right. & \in \widehat{D}^{*} F_{\bar{p}}(x, y)\left(y^{*}\right), x \in B_{\delta}(\bar{x}) \backslash F_{\bar{p}}^{-1}(\bar{y}), \\
y & \left.\in F_{\bar{p}}(x) \cap B_{\delta}(\bar{y}),\left\|y^{*}\right\|=1, \mid\left\langle y^{*}, y\right\rangle-\|y\|<\delta\right\} .
\end{aligned}
$$

Theorem 3.1 Let $X$ and $Y$ be Asplund spaces, $P$ be a metric space, and let $F$ : $P \times X \rightrightarrows Y$ be a multifunction. Consider $(\bar{p}, \bar{x}) \in P \times X$ such that $0 \in F(\bar{p}, \bar{x})$. Suppose that the following conditions hold true: 
(i) $\operatorname{gph} F_{\bar{p}}$ is closed around $(\bar{x}, 0)$, i.e., there exists $\delta>0$ such that the set $\operatorname{gph} F_{\bar{p}} \cap\left[(\bar{x}, 0)+\delta\left(B_{X} \times B_{Y}\right)\right]$ is closed;

(ii) there exist $\ell>0$ and $\delta>0$ such that

$$
F(p, x) \subset F(\bar{p}, x)+\ell d(p, \bar{p}) B_{Y} \quad \forall x \in B_{\delta}(\bar{x}), \forall p \in B_{\delta}(\bar{p})
$$

(iii) $c\left[F_{\bar{p}}\right](\bar{x}, 0)>0$.

Then for any positive $c<c\left[F_{\bar{p}}\right](\bar{x}, 0)$, the implicit multifunction $G$ defined in $(3.1)$ is calm at $(\bar{p}, \bar{x})$ with modulus $\ell / c$.

Proof. Let $\delta>0$ and $\ell>0$ be as stated in the assumptions of the theorem and let $0<c<c^{\prime}<c\left[F_{\bar{p}}\right](\bar{x}, 0)$. Based on definition (3.2), we assume without loss of generality that

$$
\left.\begin{array}{c}
x \in B_{\delta}(\bar{x}), 0 \notin F_{\bar{p}}(x), y \in F_{\bar{p}}(x) \cap \delta B_{Y}, \\
\left\|y^{*}\right\|=1,\left|\left\langle y^{*}, y\right\rangle-\|y\|\right|<\delta, x^{*} \in \widehat{D}^{*} F_{\bar{p}}(x, y)\left(y^{*}\right)
\end{array}\right\} \Rightarrow\left\|x^{*}\right\|>c^{\prime}
$$

Choose a positive $\rho<\min \{1 / 2, c /(2 \ell), 1 / \ell\} \delta$. We are going to show that

$$
G(p) \cap B_{\rho}(\bar{x}) \subset G(\bar{p})+\frac{\ell}{c} d(p, \bar{p}) B_{X} \quad \text { if } d(p, \bar{p})<\rho .
$$

Take any $p \in P$ satisfying

$$
d(p, \bar{p})<\rho
$$

and any $z \in G(p) \cap B_{\rho}(\bar{x})$. Then $p \in B_{\delta}(\bar{p}), z \in B_{\delta}(\bar{x}), 0 \in F(p, z)$. To prove the assertion we need to find a point $\hat{x} \in G(\bar{p})$ such that

$$
\|\hat{x}-z\| \leq(\ell / c) d(p, \bar{p})
$$

It follows from (3.3) that there exists $v \in F(\bar{p}, z)$ such that

$$
\|v\| \leq \ell d(p, \bar{p})
$$

Consider the l.s.c. on $(\bar{x}, 0)+\delta\left(B_{X} \times B_{Y}\right)$ function $w: X \times Y \rightarrow \mathbb{R} \cup\{\infty\}$ defined by

$$
w(x, y):= \begin{cases}\|y\|, & \text { if } y \in F_{\bar{p}}(x) \\ \infty, & \text { otherwise. }\end{cases}
$$

Obviously, $w(x, y) \geq 0$ for all $x, y$ and $w(z, v)=\|v\| \leq \ell d(p, \bar{p})$. We are going to apply the Ekeland variational principle (Lemma 2.2). The product space $X \times Y$ will 
be considered with the equivalent norm $\|(x, y)\|_{\alpha}:=\max \{\|x\|, \alpha\|y\|\}$ depending on a small parameter $\alpha>0$ such that

$$
\alpha<\min \left\{\frac{1}{c}-\frac{1}{c^{\prime}}, \frac{1}{2 c}\right\}
$$

By Lemma 2.2, there is some $(\hat{x}, \hat{y}) \in B_{\delta}(\bar{x}, 0)$ such that

$$
\begin{aligned}
& w(\hat{x}, \hat{y}) \leq w(z, v), \\
& \|(\hat{x}, \hat{y})-(z, v)\|_{\alpha} \leq \frac{\ell}{c} d(p, \bar{p}), \\
& w(\hat{x}, \hat{y}) \leq w(x, y)+c\|(x, y)-(\hat{x}, \hat{y})\|_{\alpha}, \quad \forall(x, y) \in(\bar{x}, 0)+\delta\left(B_{X} \times B_{Y}\right) .
\end{aligned}
$$

Recalling the definition of $w$, we have $\hat{y} \in F_{\bar{p}}(\hat{x})$, and conditions (3.9) and (3.11) take the following form:

$$
\begin{aligned}
& \|\hat{y}\| \leq\|v\| \\
& \|\hat{y}\| \leq\|y\|+c\|(x, y)-(\hat{x}, \hat{y})\|_{\alpha}, \forall(x, y) \in \operatorname{gph} F_{\bar{p}} \cap\left[(\bar{x}, 0)+\delta\left(B_{X} \times B_{Y}\right)\right] .
\end{aligned}
$$

Now we are going to show that $\hat{x}$ is the sought point. Due to (3.10), it satisfies (3.6), and we only need to show that $0 \in F_{\bar{p}}(\hat{x})$. Assume, to the contrary, that $0 \notin F_{\bar{p}}(\hat{x})$. Then $\hat{y} \neq 0$. Due to (3.5), (3.7), (3.10) and (3.12),

$$
\|\hat{x}-\bar{x}\|<\frac{\ell}{c} \rho+\rho<\frac{\delta}{2}+\frac{\delta}{2}=\delta, \quad\|\hat{y}\|<\ell \rho<\delta .
$$

It follows from $(3.13)$ that $(\hat{x}, \hat{y})$ is a point of local minimum of the function $\psi_{1}+$ $\psi_{2}+\psi_{3}$, where

$$
\begin{aligned}
& \psi_{1}(x, y):=\|y\|, \quad \psi_{2}(x, y):=c\|(x, y)-(\hat{x}, \hat{y})\|_{\alpha}, \\
& \psi_{3}(x, y):= \begin{cases}0, & \text { if }(x, y) \in \operatorname{gph} F_{\bar{p}}, \\
\infty, & \text { otherwise. }\end{cases}
\end{aligned}
$$

Thus, $0 \in \widehat{\partial}\left(\psi_{1}+\psi_{2}+\psi_{3}\right)(\hat{x}, \hat{y})$. Functions $\psi_{1}$ and $\psi_{2}$ are convex and Lipschitz continuous. One can apply the fuzzy sum rule (Lemma 2.1). For any $\varepsilon>0$ there exist points $\left(x_{1}, y_{1}\right),\left(x_{2}, y_{2}\right) \in X \times Y,\left(x_{3}, y_{3}\right) \in \operatorname{gph} F_{\bar{p}}$ and elements $\left(x_{1}^{*}, y_{1}^{*}\right) \in$ $\partial \psi_{1}\left(x_{1}, y_{1}\right),\left(x_{2}^{*}, y_{2}^{*}\right) \in \partial \psi_{2}\left(x_{2}, y_{2}\right),\left(x_{3}^{*}, y_{3}^{*}\right) \in \widehat{\partial} \psi_{3}\left(x_{3}, y_{3}\right)$ such that

$$
\begin{gathered}
\left\|x_{i}-\hat{x}\right\|<\varepsilon, \quad\left\|y_{i}-\hat{y}\right\|<\varepsilon, \quad i=1,2,3 \\
\left\|x_{1}^{*}+x_{2}^{*}+x_{3}^{*}\right\|<\varepsilon, \quad\left\|y_{1}^{*}+y_{2}^{*}+y_{3}^{*}\right\|<\varepsilon .
\end{gathered}
$$


By definitions of functions $\psi_{1}, \psi_{2}$, and $\psi_{3}$, we have

$$
\begin{aligned}
& x_{1}^{*}=0, y_{1}^{*} \in \partial\|\cdot\|_{Y}\left(y_{1}\right), \\
& \left\|x_{2}^{*}\right\|+\frac{1}{\alpha}\left\|y_{2}^{*}\right\| \leq c, \\
& \left(x_{3}^{*}, y_{3}^{*}\right) \in \widehat{N}\left(\left(x_{3}, y_{3}\right) ; g p h F_{\bar{p}}\right) .
\end{aligned}
$$

If $\varepsilon$ is small enough, then, due to (3.15), $y_{1} \neq 0$ and $y_{3} \neq 0$. It follows from (3.17)

$$
\left\|y_{1}^{*}\right\|=1, \quad\left\langle y_{1}^{*}, y_{1}\right\rangle=\left\|y_{1}\right\| .
$$

Taking a smaller $\varepsilon$ if necessary, we can ensure that $\varepsilon<1-c \alpha$ and, by (3.14), (3.15), and (3.17), that $x_{3} \in B_{\delta}(\bar{x}),\left\|y_{3}\right\|<\delta$. It follows from (3.16), (3.17), (3.18), and (3.20) that

$$
\begin{aligned}
& \left\|x_{3}^{*}||<c+\varepsilon, \quad||\left|y_{3}^{*} \|-1\right|<c \alpha+\varepsilon\right. \\
& \left|\left\langle y_{3}^{*}, y_{3}\right\rangle+\left\|y_{3}\left|\left\||=|\left\langle y_{1}^{*}+y_{3}^{*}, y_{3}\right\rangle+\left\langle y_{1}^{*}, y_{1}-y_{3}\right\rangle-\right\| y_{1}\right|\left|+\| y_{3}\right|||\right.\right. \\
& \leq \| y_{1}^{*}+y_{3}^{*}|| \cdot|| y_{3}||+|| y_{1}-y_{3}||+|| y_{3}||-|| y_{1}|| \mid<(c \alpha+\varepsilon) \delta+4 \varepsilon .
\end{aligned}
$$

By (3.21), $y_{3}^{*} \neq 0$ and we can define $x^{*}:=x_{3}^{*} /\left\|y_{3}^{*}\right\|, y^{*}=-y_{3}^{*} /\left\|y_{3}^{*}\right\|$. Then, by (3.19), $x^{*} \in \widehat{D}^{*} F_{\bar{p}}\left(x_{3}, y_{3}\right)\left(y^{*}\right)$ and it follows from (3.21), (3.22) that

$$
\begin{aligned}
& \left\|x^{*}\right\|<\frac{c+\varepsilon}{1-c \alpha-\varepsilon}, \quad\left\|y^{*}\right\|=1 \\
& \left|\left\langle y^{*}, y_{3}\right\rangle-\left\|y_{3}|| \leq\left|\left\langle y_{3}^{*}, y_{3}\right\rangle+\left\|y_{3}||+||\left|y_{3}^{*}\right||-1|\right\| y_{3}\right| \mid<2(c \alpha+\varepsilon) \delta+4 \varepsilon .\right.\right.
\end{aligned}
$$

By (3.8),

$$
\frac{c}{1-c \alpha}=\frac{1}{1 / c-\alpha}<c^{\prime}, \quad 2 c \alpha<1 .
$$

Taking a smaller $\varepsilon$ again, we can ensure that

$$
\frac{c+\varepsilon}{1-c \alpha-\varepsilon}<c^{\prime}, \quad 2(c \alpha+\varepsilon) \delta+4 \varepsilon<\delta .
$$

Then (3.23), (3.24) imply

$$
\left\|x^{*}\right\|<c^{\prime}, \quad\left\|y^{*}\right\|=1, \quad\left|\left\langle y^{*}, y_{3}\right\rangle-\left\|y_{3}\right\|\right|<\delta .
$$

This contradicts (3.4). Hence $0 \in F_{\bar{p}}(\hat{x})$. The proof is complete.

When applying Theorem 3.1, it is important to be able to verify the regularity assumption (iii), i.e., compute constant (3.2). We consider now some special cases when the computation of this constant can be simplified. For simplicity we limit ourselves to the case $\bar{y}=0$ which is needed in Theorem 3.1 . 
Consider a multifunction $\Pi: X \rightrightarrows Y$ defined by

$$
\Pi(x):=\left\{y \in F_{\bar{p}}(x) \mid\|y\|=\operatorname{dist}\left(0, F_{\bar{p}}(x)\right)\right\}
$$

(the projection of 0 on $F_{\bar{p}}(x)$ ). The next assertion follows directly from the definitions.

Proposition 3.2 If $\Pi(x) \neq \emptyset$ for all $x$ near $\bar{x}$ then

$$
\begin{array}{r}
c\left[F_{\bar{p}}\right](\bar{x}, 0):=\lim _{\delta \downarrow 0} \inf \left\{\left\|x^{*}\right\| \mid x^{*} \in \widehat{D}^{*} F_{\bar{p}}(x, y)\left(y^{*}\right), x \in B_{\delta}(\bar{x}) \backslash F_{\bar{p}}^{-1}(0),\right. \\
\left.y \in \Pi(x) \cap \delta B,\left\|y^{*}\right\|=1,\left\langle y^{*}, y\right\rangle=\|y\|\right\} .
\end{array}
$$

The assumption of Proposition 3.2 is satisfied automatically if $\operatorname{dim} Y<\infty$ and $F_{\bar{p}}$ is closed-valued near $\bar{x}$. In infinite dimensions it is a strong assumption, which is insured, for instance, if $\Pi$ is inner semicompact [24] at $\bar{x}$. Another (in general stronger!) regularity assumption for Theorem 3.1 can be formulated if one replaces the Fréchet coderivative of $F_{\bar{p}}$ in (3.2) (or (3.25)) with the limiting one.

To compute (3.2) or (3.25) one needs to know Fréchet coderivatives of $F_{\bar{p}}$ at all points $(x, y)$ with $x \notin F_{\bar{p}}^{-1}(\bar{y})$ near $(\bar{x}, \bar{y})$. In many situations it is preferable to deal with "at the point" conditions. It would be natural to try to replace a family of Fréchet coderivatives in definition (3.2) with a single limiting object. The outer coderivative (2.5) seems to be a good candidate. Alongside (3.2) consider another constant:

$$
c_{2}\left[F_{\bar{p}}\right](\bar{x}, \bar{y}):=\inf \left\{\left\|x^{*}\right\| \mid x^{*} \in D^{*>} F_{\bar{p}}(\bar{x}, \bar{y})\left(y^{*}\right),\left\|y^{*}\right\|=1\right\} .
$$

Proposition 3.3 Suppose $F_{\bar{p}}^{-1}$ is partially outer sequentially normally compact at $(\bar{y}, \bar{x})$. If $c_{2}\left[F_{\bar{p}}\right](\bar{x}, \bar{y})>0$ then $c\left[F_{\bar{p}}\right](\bar{x}, \bar{y})>0$.

Proof. Let $c\left[F_{\bar{p}}\right](\bar{x}, \bar{y})=0$. By (3.2), there exists sequences $\left(x_{k}, y_{k}\right) \in \operatorname{gph} F_{\bar{p}}$, $\left(x_{k}^{*}, y_{k}^{*}\right) \in X^{*} \times Y^{*}$ satisfying $x_{k} \notin F_{\bar{p}}^{-1}(\bar{y}), x_{k}^{*} \in \widehat{D}^{*} F_{\bar{p}}\left(x_{k}, y_{k}\right)\left(y_{k}^{*}\right),\left\|y_{k}^{*}\right\|=1$, $k=1,2, \ldots$, and $\left\|x_{k}^{*}\right\| \rightarrow 0,\left\langle y_{k}^{*}, \bar{y}\right\rangle \rightarrow\|\bar{y}\|$ as $k \rightarrow \infty$. Without loss of generality $y_{k}^{*} \stackrel{w^{*}}{\longrightarrow} y^{*} \in Y^{*}$. Then by definition (2.5), $0 \in D^{*>} F_{\bar{p}}(\bar{x}, \bar{y})\left(y^{*}\right)$. If $y^{*}=0$ then, due to the assumption that $F_{\bar{p}}^{-1}$ is partially outer sequentially normally compact at $(\bar{y}, \bar{x})$, we would have $\left\|y_{k}^{*}\right\| \rightarrow 0$, a contradiction. Hence, $y^{*} \neq 0$, and it follows from (3.26) that $c_{2}\left[F_{\bar{p}}\right](\bar{x}, \bar{y})=0$.

Proposition 3.4 The conclusion of Theorem 3.1 remains valid if its assumption (iii) is replaced by the following one:

(iii') $F_{\bar{p}}^{-1}$ is partially outer sequentially normally compact at $(0, \bar{x})$ and $c_{2}\left[F_{\bar{p}}\right](\bar{x}, 0)>0$. 
We next give sufficient conditions for the efficient solution map $\mathcal{S}$ defined in (1.3) to be calm at a reference point. Observe first that this efficient solution map $\mathcal{S}$ can be rewritten in the implicit multifunction form

$$
\mathcal{S}(p):=\{x \in X \mid 0 \in H(p, x)\}
$$

where $H: P \times X \rightrightarrows Y$ is a multifunction constructed via the objective function $f$ and the efficient point multifunction defined in (1.2):

$$
H(p, x):=-f(p, x)+\mathcal{F}(p) .
$$

For the sake of clarity we shall use the notation $H_{p}:=H(p, \cdot)$.

Theorem 3.5 Let $X$ and $Y$ be Asplund spaces, $P$ be a metric space and let multifunction $H$ be defined by (3.28). Consider $(\bar{p}, \bar{x}) \in$ gphS . Suppose that the following conditions hold true:

(i) $\operatorname{gph} H_{\bar{p}}$ is closed around $(\bar{x}, 0)$, i.e., there exists $\delta>0$ such that the set $\operatorname{gph} H_{\bar{p}} \cap\left[(\bar{x}, 0)+\delta\left(B_{X} \times B_{Y}\right)\right]$ is closed;

(ii) there exist $\ell>0$ and $\delta>0$ such that

$$
H(p, x) \subset H(\bar{p}, x)+\ell d(p, \bar{p}) B_{Y} \quad \forall x \in B_{\delta}(\bar{x}), \forall p \in B_{\delta}(\bar{p})
$$

(iii) $c\left[H_{\bar{p}}\right](\bar{x}, 0)>0$.

Then for any positive $c<c\left[H_{\bar{p}}\right](\bar{x}, 0)$ the efficient solution map $\mathcal{S}$ of (1.1) is calm at $(\bar{p}, \bar{x})$ with modulus $\ell / c$.

Proof. The proof is directly derived from Theorem 3.1 by considering the multifunction $H$ instead of $F$.

Proposition 3.6 The conclusion of Theorem 3.5 remains valid if its assumption (iii) is replaced by the following one:

(iii') $H_{\bar{p}}^{-1}$ is partially outer sequentially normally compact at $(0, \bar{x})$ and $c_{2}\left[H_{\bar{p}}\right](\bar{x}, 0)>0$.

Remark 3.7 It is easy to verify that the property of $H$ declared in Theorem 3.5 (ii) is ensured by the following two conditions:

(a) there exist neighborhoods $U$ of $\bar{x}, W$ of $\bar{p}$, and a real number $\ell_{1}>0$ such that

$$
\|f(p, x)-f(\bar{p}, x)\| \leq \ell_{1} d(p, \bar{p}) \quad \forall x \in U, \forall p \in W
$$

(b) $\mathcal{F}$ in (1.2) is locally upper Lipschitzian at $\bar{p}$. 
The reader is referred to $[2,3]$ for several sufficient conditions ensuring the local upper Lipschitzian property of $\mathcal{F}$ at the reference point.

The next example illustrates the importance of assumption (ii) in Theorem 3.5, namely the calmness of $\mathcal{S}$ defined in (1.3) may be violated if this assumption is omitted.

Example 3.8 Let $P=X=Y=\mathbb{R}, K=\mathbb{R}_{+}:=[0 ;+\infty)$ and let $f: \mathbb{R} \times \mathbb{R} \rightarrow \mathbb{R}$ be a function given as follows:

$$
f(p, x)= \begin{cases}|x-p|+p, & \text { if } p<0, x \in \mathbb{R}, \\ |x-\sqrt{p}|, & \text { if } p \geq 0, x \in \mathbb{R} .\end{cases}
$$

We consider the problem (1.1) with the efficient solution map $\mathcal{S}$ defined in (1.3). For $\bar{p}=0$, we have $\mathcal{F}(\bar{p})=\{0\}, \mathcal{S}(\bar{p})=\{0\}$. Let $\bar{x}=0$. Then $(\bar{p}, \bar{x}) \in \operatorname{gph} \mathcal{S}$. By simple computation, one gets

$$
H_{\bar{p}}(x)=-|x| \quad \forall x \in \mathbb{R} .
$$

So, gph $H_{\bar{p}}$ is closed. Take any $x \in \mathbb{R}$ with $0 \notin H_{\bar{p}}(x)$. The last condition means that $x \neq 0$. Set $y:=H_{\bar{p}}(x)=-|x|$. Then for any $y^{*} \in \mathbb{R}$ we have

$$
\widehat{D}^{*} H_{\bar{p}}(x, y)\left(y^{*}\right)= \begin{cases}-y^{*}, & \text { if } x>0 \\ y^{*}, & \text { if } x<0\end{cases}
$$

and it follows from (3.2) that $c\left[F_{\bar{p}}\right](\bar{x}, \bar{y})=1$. This means that condition (iii) of Theorem 3.5 is fulfilled. It is easy to see that

$$
H(p, x)=-f(p, x)+\mathcal{F}(p)= \begin{cases}-|x-p|, & \text { if } p<0, x \in \mathbb{R} \\ -|x-\sqrt{p}|, & \text { if } p \geq 0, x \in \mathbb{R}\end{cases}
$$

and thus condition (ii) of Theorem 3.5 is not satisfied. The efficient solution map

$$
\mathcal{S}(p)= \begin{cases}p, & \text { if } p<0 \\ \sqrt{p}, & \text { if } p \geq 0\end{cases}
$$

is not calm at $(\bar{p}, \bar{x})$.

The following corollary provides sufficient conditions for the efficient solution map $\mathcal{S}$ in (1.3) to be calm at the reference point under the strict differentiability of the objective function $f$. 
Corollary 3.9 Let $X$ and $Y$ be Asplund spaces, $P$ be a metric space and let multifunction $H$ be defined by (3.28). Consider $(\bar{p}, \bar{x}) \in$ gph $\mathcal{S}$. Suppose that $f$ is strictly differentiable at $(\bar{p}, \bar{x})$ and the following conditions hold true:

(i) $\operatorname{gph} H_{\bar{p}}$ is closed around $(\bar{x}, 0)$, i.e., there exists $\delta>0$ such that the set $\operatorname{gph} H_{\bar{p}} \cap\left[(\bar{x}, 0)+\delta\left(B_{X} \times B_{Y}\right)\right]$ is closed;

(ii) multifunction $\mathcal{F}$ defined in (1.2) is locally upper Lipschitzian at $\bar{p}$;

(iii) $c\left[H_{\bar{p}}\right](\bar{x}, 0)>0$.

Then the efficient solution map $\mathcal{S}$ of $(1.1)$ is calm at $(\bar{p}, \bar{x})$.

Proof. Since $f$ is strictly differentiable at $(\bar{p}, \bar{x})$, the condition (3.29) is fulfilled. This together with the local upper Lipschitzian property of $\mathcal{F}$ implies by Remark 3.7 that $H$ satisfies assumption (ii) in Theorem 3.5. The conclusion follows from this theorem.

As previously, the key issue when applying Corollary 3.9, is the computation of constants $c\left[H_{\bar{p}}\right](\bar{x}, 0)$ or $c_{2}\left[H_{\bar{p}}\right](\bar{x}, 0)$ (see Proposition 3.4$)$, which requires the knowledge of the family of Fréchet coderivatives of $H_{\bar{p}}$ at nearby points or the limiting coderivative (under the partial outer sequential normal compactness assumption). Taking into account definition (3.28) one can notice that $H_{\bar{p}}$ is the sum of the strictly differentiable function $f(\bar{p}, \cdot)$ (under the conditions of Corollary 3.9) and the constant multifunction $\mathcal{F}(\bar{p})$ (it is a set in $Y$ which does not depend on $x$ ). These observations can significantly simplify the computation. For instance, it is easy to see that in this situation the restriction $0 \notin H_{\bar{p}}(x)$ does not play any role when defining $D^{*>} H_{\bar{p}}(\bar{x}, 0)$ (see definition $(2.5)$ ). Hence,

$$
D^{*>} H_{\bar{p}}(\bar{x}, 0)\left(y^{*}\right)=D^{*} H_{\bar{p}}(\bar{x}, 0)\left(y^{*}\right) \quad \forall y^{*} \in Y^{*} .
$$

Utilizing the coderivative sum rule given in [24, Theorem 1.62 (ii)], we arrive at

$$
D^{*} H_{\bar{p}}(\bar{x}, 0)\left(y^{*}\right)=-\left(\nabla_{x} f(\bar{p}, \bar{x})\right)^{*} y^{*}+D^{*} \mathcal{F}(\bar{p})(\bar{x}, f(\bar{p}, \bar{x}))\left(y^{*}\right)
$$

where $\nabla_{x} f(\bar{p}, \bar{x})$ denotes the Fréchet derivative of $f(\bar{p}, \cdot)$ at $\bar{x}$. Taking into account that $\mathcal{F}(\bar{p})$ is a constant multifunction we have

$$
x^{*} \in D^{*} \mathcal{F}(\bar{p})(\bar{x}, f(\bar{p}, \bar{x}))\left(y^{*}\right) \quad \Leftrightarrow \quad x^{*}=0,-y^{*} \in N(f(\bar{p}, \bar{x}) ; \mathcal{F}(\bar{p})),
$$

and consequently,

$$
c_{2}\left[H_{\bar{p}}\right](\bar{x}, 0)=\inf \left\{\left\|\left(\nabla_{x} f(\bar{p}, \bar{x})\right)^{*} y^{*}\right\| \mid y^{*} \in N(f(\bar{p}, \bar{x}) ; \mathcal{F}(\bar{p})),\left\|y^{*}\right\|=1\right\} .
$$


It is not difficult to establish a similar representation for constant $c\left[H_{\bar{p}}\right](\bar{x}, 0)$. For this purpose a "fuzzy" analog of [24, Theorem 1.62 (ii)] is needed which can be established along the lines of [20, Proposition 1.6]

We finish this section by a example which is designed to show how Theorem 3.5 can be applied to concrete problems.

Example 3.10 Let $P=X=\mathbb{R}, Y=\mathbb{R}^{2}, K=\mathbb{R}_{+}^{2}$, let $\mathbb{R}^{2}$ be equipped with the Euclidean norm, and let $f: \mathbb{R} \times \mathbb{R} \rightarrow \mathbb{R}^{2}$ be given as follows:

$$
f(p, x)=(|p-x|+p,|p-x|+p) \quad \forall x \in \mathbb{R}, \forall p \in \mathbb{R} .
$$

We consider the problem (1.1) with the efficient solution map $\mathcal{S}$ defined in (1.3). For $\bar{p}=0$, we have $\mathcal{F}(\bar{p})=\{(0,0)\}, \mathcal{S}(\bar{p})=\{0\}$. Let $\bar{x}=0$. Then $(\bar{p}, \bar{x}) \in$ gph $\mathcal{S}$. By simple computation, one gets

$$
H_{\bar{p}}(x)=\Pi(x)=-(|x|,|x|) \quad \forall x \in \mathbb{R} .
$$

So, gph $H_{\bar{p}}$ is closed. Take any $x \in \mathbb{R}$ with $0 \notin H_{\bar{p}}(x)$. The last condition means that $x \neq 0$. Set $y:=H_{\bar{p}}(x)=-(|x|,|x|)$ and consider $y^{*}:=\left(y_{1}^{*}, y_{2}^{*}\right) \in \mathbb{R}^{2}$ satisfying $\left\langle y^{*}, y\right\rangle=\|y\|$. Obviously, $y_{1}^{*}+y_{2}^{*}=-\sqrt{2}$, and we have

$$
\widehat{D}^{*} H_{\bar{p}}(x, y)\left(y^{*}\right)= \begin{cases}-y_{1}^{*}-y_{2}^{*}=\sqrt{2}, & \text { if } x>0 \\ y_{1}^{*}+y_{2}^{*}=-\sqrt{2}, & \text { if } x<0 .\end{cases}
$$

Condition $\left\|y^{*}\right\|=1$ can be easily satisfied if one takes $y_{1}^{*}=y_{2}^{*}=-\sqrt{2} / 2$. It follows

from $(3.25)$ that $c\left[F_{\bar{p}}\right](\bar{x}, \bar{y})=\sqrt{2}$. This means that the condition (iii) of Theorem 3.5 is fulfilled. It is easy to see that $H(p, x)=-f(p, x)+\mathcal{F}(p)=-(|p-x|,|p-x|)$ for all $p \in P$, all $x \in X$, and thus

$$
\|H(p, x)-H(0, x)\|=\sqrt{2}|| x|-| x-p|| \leq \sqrt{2}|p| \quad \forall p \in P, \forall x \in X
$$

which verifies condition (ii) of Theorem 3.5. Hence, $\mathcal{S}$ is calm at $(\bar{p}, \bar{x})$.

\section{References}

[1] E. M. Bednarczuk, Some stability results for vector optimization problems in partially ordered topological vector, in: Proc. First World Congress Nonlin. Anal., vol. III, Tampa, Florida (1996), 2371 - 2382. 
[2] E. M. Bednarczuk, Upper Hölder continuity of minimal points, J. Convex Anal., 9 (2002), no. 2, 327-338.

[3] E. M. Bednarczuk, Hölder-like properties of minimal points in vector optimization, Control Cybernet., 31 (2002), no. 3, 423-438.

[4] J. F. Bonnans, and A. Shapiro, Perturbation Analysis of Optimization Problems, Springer, New York, 2000.

[5] A. Chinchuluun, P. M. Pardalos, A survey of recent developments in multiobjective optimization, Annals of Operations Research, 154 (2007), 29-50.

[6] A. Chinchuluun, P. M. Pardalos, A. Migdalas, L. Pitsoulis (Eds.), Pareto Optimality, Game Theory and Equilibria, Springer, 2008.

[7] T. D. Chuong, N. Q. Huy and J.-C. Yao, Stability of semi-infinite vector optimization problems under functional purtubations, J. Global Optim., 45 (2009), $583-595$.

[8] T. D. Chuong, N. Q. Huy and J.-C. Yao, Pseudo-Lipschitz property of linear semi-infinite vector optimization problems, European J. Oper. Research, 200 (2010), 639-644.

[9] T. D. Chuong and J.-C. Yao, Sufficient conditions for pseudo-Lipschitz property in convex semi-infinite vector optimization problems, Nonlinear Anal., 71 (2009), 6312-6322.

[10] T. D. Chuong, J.-C. Yao and N. D. Yen, Further results on the lower semicontinuity of efficient point multifunctions, Pacific J. Optim., 6 (2010), 405-422.

[11] I. Ekeland, On the variational principle, J. Math. Anal. Appl. 47 (1974) 324353.

[12] C. A. Floudas, P. M. Pardalos, (Eds.), Encyclopedia of Optimization, Springer, 2009 .

[13] A. Gopfert, H. Riahi, C. Tammer and C. Zălinescu, Variational Methods in Partially Ordered Spaces, Springer, New York, 2003.

[14] R. Henrion and A. Jourani, Subdifferential conditions for calmness of convex constraints, SIAM J. Optim., 13 (2002), 520-534. 
[15] R. Henrion, A. Jourani, and J. Outrata, On the calmness of a class of multifunctions, SIAM J. Optim., 13 (2002), 603-618.

[16] R. Henrion and J. Outrata, Calmness of constraint systems with applications, Math. Program., 104 (2005), 437-464.

[17] N. Q. Huy and J.-C. Yao, Stability of implicit multifunctions in Asplund spaces, Taiwan. J. Math., 13 (2009), 47-65.

[18] A. D. Ioffe and J. Outrata, On metric and calmness qualification conditions in subdifferential calculus. Set-Valued Anal. 16, (2008), no. 2-3, 199-227.

[19] D. Klatte and B. Kummer, Nonsmooth Equations in Optimization. Regularity, Calculus, Methods and Applications. Kluwer Acad. Publ., Dordrecht-BostonLondon, 2002.

[20] A. Y. Kruger, On Fréchet subdifferentials. J. Math. Sci. (N. Y.) 116, (2003), no. 3, 3325-3358.

[21] Y. S. Ledyaev and Q. J. Zhu, Implicit multifunctions theorems, Set-Valued Anal., 7 (1999), 209-238.

[22] G. M. Lee, N. N. Tam and N. D. Yen, Normal coderivative for multifunctions and implicit function theorems, J. Math. Anal. Appl., 338 (2008), 11-22.

[23] D. T. Luc, Theory of Vector Optimization. Lect. Notes Econ. Math. Syst., 319, Springer, Berlin, 1989.

[24] B. S. Mordukhovich, Variational Analysis and Generalized Differentiation. I: Basic Theory, Springer, Berlin, 2006.

[25] R. R. Phelps, Convex Functions, Monotone Operators and Differentiability, 2nd ed., Lect. Notes Math. 1364, Springer, Berlin, 1993.

[26] R. T. Rockafellar, R. J-B. Wets, Variational Analysis. Springer, Berlin, 1998.

[27] S. M. Robinson, Generalized equations and their solutions, Part I: Basic theory, Math. Program. Study, 10 (1979), 128-141.

[28] Y. Sawaragi, H. Nakayama and T. Tanino, Theory of Multiobjective Optimization. Mathematics in Science and Engineering, 176. Academic Press, Inc., Orlando, FL, 1985. 
[29] S. W. Xiang and Y. H. Zhou, Continuity properties of solutions of vector optimization, Nonlinear Anal. 64 (2006), 2496-2506.

[30] S. W. Xiang and W. S. Yin, Stability results for efficient solutions of vector optimization problems, J. Optim. Theory Appl, 134 (2007), 385-398.

[31] X. Y. Zheng and Kung Fu Ng, Metric subregularity and constraint qualifications for convex generalized equations in Banach spaces, SIAM J. Optim. 18 (2007), no. $2,437-460$.

[32] X. Y. Zheng and Kung Fu Ng, Calmness for L-subsmooth multifunctions in Banach spaces, SIAM J. Optim. 19 (2009), no. 4, 1648-1673. 